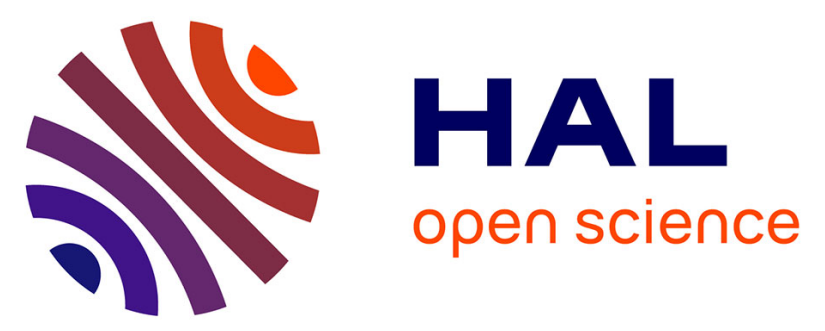

\title{
Recensement et analyse des outils examinant la qualité de la relation fraternelle: Recherche-action menée auprès de SOS Village d'Enfants
}

Chantal Zaouche Gaudron, S. Pinel-Jacquemin, Olivia Troupel-Cremel, R. Scelles

\section{To cite this version:}

Chantal Zaouche Gaudron, S. Pinel-Jacquemin, Olivia Troupel-Cremel, R. Scelles. Recensement et analyse des outils examinant la qualité de la relation fraternelle: Recherche-action menée auprès de SOS Village d'Enfants. Pratiques Psychologiques, 2016, 22 (4), pp.363-380. 10.1016/j.prps.2016.03.002 . hal-01498730

\section{HAL Id: hal-01498730 \\ https://hal-univ-tlse2.archives-ouvertes.fr/hal-01498730}

Submitted on 30 Mar 2017

HAL is a multi-disciplinary open access archive for the deposit and dissemination of scientific research documents, whether they are published or not. The documents may come from teaching and research institutions in France or abroad, or from public or private research centers.
L'archive ouverte pluridisciplinaire HAL, est destinée au dépôt et à la diffusion de documents scientifiques de niveau recherche, publiés ou non, émanant des établissements d'enseignement et de recherche français ou étrangers, des laboratoires publics ou privés. 
Psychologiques

Elsevier Editorial system(tm) for Pratiques

Manuscript Draft

Manuscript Number: PRPS-D-15-00057R1

Title: Recensement et analyse des outils examinant la qualité de la relation fraternelle

Article Type: Psychologie du developpement

Keywords: fratrie; relations fraternelles; outils; évaluation; rechercheaction

Corresponding Author: Madame Chantal Zaouche,

Corresponding Author's Institution: UTM, MDR

First Author: Chantal Zaouche

Order of Authors: Chantal Zaouche; Stéphanie PINEL-JACQUEMIN, Dr.; Olivia TROUPEL, Dr.; Régine SCELLES, Pr. 


\section{Recensement et analyse des outils examinant la qualité de la relation fraternelle ${ }^{1}$ Inventory and analysis of tools examining the quality of sibling relationships}

Zaouche Gaudron, Chantal ${ }^{* 2}$, Pinel-Jacquemin, Stéphanie ${ }^{3}$, Troupel-Cremel, Olivia ${ }^{4} \&$ Scelles, Régine ${ }^{5}$

* Chantal.zaouche@univ-tlse2.fr

Université Toulouse Jean Jaurès UMR LISST-CERS

Bureau 330, 5 allées A. Machado

31058 Toulouse Cedex 9

Tel : 0561502581

Fax : 0561503870

${ }^{1}$ Recherche financée par l'ONED

${ }^{2}$ Professeure de Psychologie de l'enfant, Université Toulouse Jean Jaurès

${ }^{3}$ Post-doctorante en psychologie, Université Toulouse Jean Jaurès

${ }^{4}$ Maitre de conférences en Psychologie du développement, Université Toulouse Jean Jaurès

${ }^{5}$ Professeure de Psychologie, Université de Rouen 


\begin{abstract}
Résumé
La loi française de 1996 prévoit dans le code civil le maintien de la communauté de vie des enfants sauf si des motifs graves sont avérés. Pourtant, peu d'outils sont à disposition des professionnels de l'enfance pour leur permettre d'évaluer la qualité des relations fraternelles. Pour répondre à ce besoin, cette étude a d'abord répertorié 21 outils concernant l'évaluation des liens fraternels, les chercheurs en ont, ensuite, sélectionné 8 , couvrant la petite enfance, l'enfance et l'adolescence, et ont demandé à 12 psychologues des Villages SOS de les tester. Cette recherche-action a aussi eu pour visée de contribuer à la prise en considération de la dimension fraternelle par les professionnels des villages SOS Villages d'Enfants.
\end{abstract}

Mots-clés : fratrie, relations fraternelles, outils, évaluation, recherche-action

\begin{abstract}
The French law of 1996 plans in the civil code the preservation, except grave motives, of the community of life of the children. Nevertheless few tools are available for the professionals of the childhood to allow them to estimate objectively the quality of the sibling relationships. In order to meet this need, this study listed, at first, the available search tools concerning the evaluation of the sibling links. Researchers retained then 8 of them, covering the toddler, childhood and adolescence periods, and asked 12 psychologists to test them.
\end{abstract}


This research-action also had for aim to contribute in a wider way to the consideration of the sibling dimension by the professionals of SOS children Villages.

Keywords: sibling, sibships, instruments, assessment, research-action

\section{Conflit d'intérêt : aucun}




\section{Recensement et analyse des outils examinant la qualité de la relation \\ fraternelle \\ Recherche-action menée auprès de SOS Village d'Enfants}

\section{Introduction}

Dans les cas où des enfants ne peuvent pas vivre avec leurs parents, il s'agit de savoir si les frères et sœurs doivent être accueillis au sein d'une même famille ou d'une même institution ou doivent être séparés. En effet, à l'heure actuelle, et dans notre pays, deux postures coexistent. La première tend à maintenir la fratrie ensemble «à tout prix » quel que soit le lien qui s'est construit et existe entre frères et sœurs et s'appuie notamment sur la loi de 1996. La seconde, plus récente, pose la question de la qualité de la relation fraternelle et des processus qui permettent de la préciser. Il s'agit d'évaluer la qualité de ce lien et comment la définir sachant que certains processus tels que la jalousie, la rivalité, processus constitutifs de cette relation, ne permettent pas à eux seuls de la qualifier de «bonne » ou de «mauvaise », et surtout de valider si ce lien est source de soutien ou de souffrance (Troupel, Pinel-Jacquemin \& Zaocuhe Gaudron, 2009).

Par ailleurs, les nouvelles configurations familiales (notamment les recompositions avec les demi/quasis frères amènent à creuser la question de ce qu'est en soi une fratrie : avoir uniquement des liens de sang ? Vivre au quotidien ensemble? Etre sous le même toit un temps suffisamment conséquent pour que des liens affectifs se forgent? 
Dans le contexte de l'aide sociale à l'enfance, en particulier quand les enfants doivent être séparés de leurs parents et placés ensemble, il est fondamental de pouvoir comprendre si la relation fraternelle est un étai, une source de protection et de sécurisation pour eux ou si elle est une source de souffrance (la maltraitance intrafraternelle étant l'illustration la plus saillante). Il s'agit aussi d'anticiper le fait que la vie quotidienne des enfants dans les établissements ne contribue pas à perpétuer les relations familiales dysfonctionnelles.

Confrontée à cette question, l'Aide Sociale à l'Enfance (ASE), en France, doit prendre la mesure des risques et des bénéfices du placement fraternel conjoint. C'est dans ce contexte que l'association SOS Villages d'Enfants, dont la spécificité est d'accueillir, sur une longue durée, les fratries confiées par l'ASE, a souhaité qu'une recherche-action soit menée. L'objectif était de mieux cerner les intérêts et les limites du placement conjoint des fratries. Pour cela, l'association voulait savoir s'il serait possible que les psychologues des villages disposent d'un outil permettant d'évaluer la qualité des liens fraternels pour éclairer la décision à prendre lors de l'admission et en cours de placement.

Cette recherche-action avait aussi pour visée de sensibiliser les professionnels au travail de recherche et à la possibilité et/ou la nécessité d'une évaluation rigoureuse et objective des liens fraternels. En effet, une première étude, financée par SOS Village d'Enfants et la Fondation de France, avait permis de conclure au manque d'outils d'évaluation spécifiques et adaptés au travail avec les fratries (Pinel-Jacquemin, Chéron, Favart, Dayan \& Scelles, 2013; Scelles, Favart, Chéron \& Pinel-Jacquemin, 2008). 


\footnotetext{
${ }^{1}$ Cette étude a été réalisée en partenariat avec SOS Villages d'Enfants. Depuis plus de 50 ans, cette association développe un modèle de prise en charge centré sur le droit de l'enfant à grandir dans un contexte de vie de type familial. Des fratries sont accueillies dans l'hypothèse d'un placement de longue durée sur demande de l'Aide Sociale à l'Enfance.
}

\begin{abstract}
Afin de pallier ce manque, le premier objectif de cette recherche-action a été de recenser et d'analyser les outils utiles à l'évaluation de la qualité des relations fraternelles et qui prennent en compte le point de vue de l'enfant (Zaouche Gaudron, Troupel-Cremel, \& Pinel-Jacquemin, 2010) ${ }^{1}$. Il s'agissait de comprendre via l'évaluation de psychologues si les outils sélectionnés par les chercheurs pouvaient permettre, d'une part, d'identifier les ressources et compétences fraternelles sur lesquelles les enfants pouvaient s'appuyer, et d'autre part, de déceler et d'analyser d'éventuels dysfonctionnements de ces liens aux effets délétères pouvant nuire au bien-être et au développement psycho-affectif des enfants. Le deuxième objectif était à la fois d'informer et de former les psychologues à la démarche de recherche et à l'utilisation d'outils dans une démarche active durant laquelle, ils devaient en valider, ou non, la pertinence.

Pour répondre à cette démarche de recherche-action, la méthodologie s'est déroulée en trois temps: un temps d'information et de formation auprès des psychologues des 12 villages SOS du territoire national, un temps de passation des outils et un temps de retour sur expérience et d'évaluation menée de manière standardisée.
\end{abstract}

\title{
1. Lien fraternel : fonctionnel versus dysfonctionnel
}


Les relations au sein de la fratrie ont pendant longtemps constitué un sujet de recherche négligé par les spécialistes du développement de l'enfant. Aujourd'hui, elles sont étudiées avec diverses approches dont les principales sont les théories développementales, systémique et psychanalytique. Elles s'attachent à décrire à la suite de Adler (1920/1958), le groupe (taille, écart d'âge, sexe) pour inférer l'impact du lien sur chacun de ses membres (Bourguignon, 1999 ; Dunn, 1983). Le groupe fraternel opère comme un transformateur de la vie psychique familiale et la relation fraternelle interne va dépendre, selon Vinay et Jayle (2011), des liens horizontaux et de l'imbrication de ceux-ci avec les liens parents-enfants. Depuis quelques années cependant, un certain nombre d'études ont été entreprises, afin de mieux connaître les caractéristiques propres aux relations fraternelles. Kaës (1993) souligne que le groupe fraternel est caractérisé par l'absence de différences de génération, par un effet de rang «dans l'ensemble égalitaire » (p. 24), même s'il comprend des écarts d'âges qui assignent « des emplacements qui structurent les rivalités, les soumissions » (p. 24), et enfin par des «repères identificatoires symboliques (...) qui inscrivent l'enfant dans une lignée et un ensemble synchrone » (p. 24). D'abord étudié comme dépendant du couple parental par les auteurs d'obédience psychanalytique (Freud, 1922; Rabain, 1995), le groupe fraternel est à présent reconnu comme ayant ses règles et sa dynamique propre (Kaës, 1993 ; Bourguignon, 1999 ; Jaïtin, 2006). L'approche systémique, quant à elle, considère la fratrie comme un sous-groupe de la famille qui se construit et évolue au cours du cycle de la vie des familles dans un jeu d'interdépendances avec les différents sous-systèmes familiaux 
(intergénérationnel, conjugal, coparental, parent-enfant) (Minuchin, 1979 ; Tilmans-Ostyn \& Meynckens-Fourez, 1999). Au sein de la famille, entre enfants, entre enfants et parents, entre chaque enfant et chaque parent et le couple parental, se jouent des phénomènes complexes d'identifications, de différenciations, d'amour, de haine, de rivalité, de jalousie... Etre et surtout devenir à la fois «même» et «différent» est le cœur de la problématique fraternelle (Bourguignon, 1999). L'expérience de ces processus contribue à développer chez l'enfant l'empathie et la bienveillance, l'identification rendant l'autre familier et la différenciation permettant la construction d'une altérité non menaçante et source de désir (Graham-Bermann \& Cutler, 1994 ; Scelles, 2010).

Les fonctions de la fratrie dans la construction identitaire, dans le processus d'apprentissage des compétences sociales, de la gestion de sa propre agressivité et de celle de l'autre, le lien qu'elle entretient avec l'histoire du sujet, fait qu'une fratrie fonctionnelle peut être une ressource pour les enfants en situation de placement (Troupel, Pinel-Jacquemin \& Zaouche Gaudron, 2009). Le clan formé peut être sécurisant, donner un sentiment de force, parce qu'il instaure proximité, cohérence, complétude, lien au groupe dont il est issu, même si, parfois, il peut aussi s'avérer aliénant.

Fine (2007) souligne deux dangers potentiels existant au sein de la relation fraternelle. Le premier serait l'absence d'amour et/ou de solidarité, qui peut aller jusqu'au fratricide (dont les contes et légendes sont friands). Le second serait l'excès d'amour qui, lui, peut mener à une relation incestueuse. L'idéal situerait la fraternité comme lien désexualisé, à connotation affective. 
Si l'ambivalence est au cœur même de la dynamique de ce lien entre amour et rivalités ${ }^{2}$, les conflits entre frères et sœurs peuvent s'avérer destructeurs, et il importe de pouvoir évaluer le seuil au-delà duquel ils peuvent se transformer en violence, voire en maltraitance (Pinel-Jacquemin \& Scelles, 2012). Benarous, Consoli, Raffin et Cohen $(2014,302)$ notent que « la maltraitance limitée à un enfant de la fratrie concerne $44 \%$ des enfants exposés à la maltraitance ». Les conséquences des violences intrafamiliales peuvent être modérées par les relations fraternelles soutenantes (Troupel-Cremel, Pinel-Jacquemin \& Zaouche Gaudron, 2009), par la chaleur de la relation et du contexte parental (Sharifi, Jabbarifard, Rezaee, Hafshejani \& Zare, 2015). Des enjeux de pouvoir et de domination peuvent également ternir des relations fraternelles (Graham-Bermann \& Cutler, 1994).

Beauregard (2003) rapporte le fait que lorsque les interactions diminuent au sein d'un sous-groupe de la famille (celui des parents, par exemple), cela entrainerait une augmentation des interactions au sein des autres sous-groupes (la fratrie). Cette augmentation concernerait tant des aspects positifs (coopération, sollicitude, etc.) que des aspects négatifs (agressions, violences diverses...). De leur côté, Buhrmester et Furman (1990) décrivent l'effet « spillover » selon lequel les sentiments et les affects éprouvés dans l'un des sous-systèmes se propageraient dans un autre sous-système.

\footnotetext{
${ }^{2}$ Voir par exemple les titres souvent évocateurs des ouvrages traitant de la fratrie, comme « Frères et sœurs, une maladie d'amour » (Rufo, 2003), «Frères et sœurs, complices et rivaux » (Scelles, 2003), «Frères et sœurs intimes ennemis : à propos du complexe fraternel » (Bouhassira Chiron, 2012).
} 
Ces éléments montrent à quel point ce qui se passe au sein du groupe fratrie est complexe, le lien fraternel pouvant être une ressource ou une source de souffrance quand, par exemple, la jalousie et l'agressivité structurante font place à des maltraitances entre enfants.

Si les maltraitances intra-fraternelles existent (Bass \& al., 2006; GrahamBermann, 1994), elles doivent être connues des personnes chargées de protéger les fratries. Or, les enfants les dénoncent rarement : le faire reviendrait à rompre doublement avec la loyauté filiale et fraternelle et serait très coûteux sur le plan psychique et physique, si le coupable dénoncé venait notamment à redoubler de violence. Par ailleurs, les familles ont quelques réticences à faire état de ces maltraitances avec les professionnels. Ils les banalisent la plupart du temps, en partie, pour se défendre de leur propre culpabilité de ne pas parvenir à instaurer un climat serein entre leurs enfants (Pinel-Jacquemin \& Scelles, 2012).

Il arrive aussi qu'un enfant battant son frère soit, en quelque sorte, le «bras armé » de l'un de ses deux parents. Il tape alors son frère comme il imagine, ou sait, que l'adulte voudrait le taper. Cette violence intra-fraternelle, quelle que soit la façon dont elle est transmise, atteint parfois de manière durable les assises narcissiques et identitaires de l'enfant victime (Angel, 1996), d'où l'importance d'avoir des outils et une méthodologie pour la repérer et l'évaluer le plus objectivement possible.

Il est également à noter que les auteurs s'accordent pour souligner le fait que dans le cas de maltraitance intra-fraternelle, par le jeu complexe d'identification/différenciation/projection/introjection, la victime et l'agresseur 
peuvent se trouver dans une situation où tous les deux sont finalement victimes et agresseurs et demandent à être aidés, soignés sans pour autant que soit déniée la réalité du statut de victime et d'agresseur. Ainsi, si l'agresseur est plus souvent un aîné qu'un cadet, un garçon qu'une fille, il s'avère que la littérature rapporte que fréquemment l'agresseur a été auparavant lui-même agressé et continue parfois à l'être. Par ailleurs, les travaux s'entendent pour rappeler qu'il est impossible de comprendre le processus d'émergence de la maltraitance entre enfants sans prendre en compte le climat familial en général, et celui entre les membres du couple en particulier (Buhrmester \& Furman, 1990). Enfin, il est important de souligner que la recherche sur le placement des enfants s'est focalisée durant de nombreuses années sur l'analyse intergénérationnelle et le devenir des enfants à l'âge adulte et très peu sur les liens fraternels. L'analyse de ces liens est le plus souvent esquissée (Tilmans-Ostyn \& Meynckens-Fourez, 1999) et est très peu abordée en France (Drapeau \& al, 2000 ; Simard \& al., 1998), si ce n'est dans des approches anthropologiques rétrospectives comme la recherche de Cadoret (1998).

\section{Protocole de recherche}

\subsection{Etapes de la recherche}

Une première étape a eu pour visée de recenser les outils qui évaluent de façon spécifique les liens fraternels. Elle a été réalisée par Scelles, Favart, Chéron et Pinel-Jacquemin $(2008)^{3}$, à partir d'une revue de la littérature internationale sur la base des mots-clés suivants : sibling, relationships, sibships, foster care, violence,

\footnotetext{
${ }^{3}$ Scelles, R., Favart, E., Chéron, J. \& Pinel-Jacquemin, S. (2008). Accueil conjoint des fratries : analyse de la littérature sciences humaines et sociales. Rapport de Septembre 2008. Etude demandée et financée par l'Association SOS Villages d'Enfants avec le soutien de la Fondation de France.
} 
agression, incest, abuse, maltreatment, placement, fratrie, liens fraternels, relations fraternelles, maltraitance, violence fraternelle, maltraitance, abus, inceste, dans les banques de données Sciencedirect, Scopus, EBSCO, CAIRN, Scirus, Sage Publications, Psycinfo, Psycarticle, Eric, Medline, BDSP et Psychological and Behavorial Sciences Collection.

La seconde étape a eu pour objectif de réaliser une analyse critique des outils recensés. Le but de l'étude n'étant pas la validation dans une version francophone des outils déjà validés en langue anglaise selon les normes en vigueur, nous avons procédé à la manière de la rétro-traduction proposée par Vallerand (1989). Dans un premier temps, les items ont été traduits en français par l'une d'entre nous. La traduction de certains termes a été l'objet d'une attention particulière afin d'être adaptée à la culture française. Selon la méthode de rétro-traduction, les items ont été ensuite retraduits en anglais. Aucune modification majeure n'a été relevée nécessitant une modification de la traduction initiale. Nous mentionnons aussi que les items sont relativement simples dans leur expression première, ce qui a facilité la méthode de rétro-traduction préconisée par Vallerand (1989).

Au total, nous avons recensé 21 outils validés scientifiquement et ayant fait l'objet d'une publication. Pour chacun d'entre eux, une fiche descriptive a été complétée à partir du modèle élaboré par Zaouche-Gaudron, Euillet et Pinel-Jacquemin $(2007)^{4}$ qui présente le descriptif de l'outil, le contexte théorique dans lequel il a été développé, ses qualités métrologiques, ses intérêts et limites.

\footnotetext{
${ }^{4}$ Zaouche-Gaudron, C., Euillet, S., \& Pinel-Jacquemin, S. (2007). Recensement critique des instruments de recherche validés en langue française en psychologie du développement : Bilan sur 10 ans (1995 à 2005). Rapport terminal, Observatoire National de l'Enfance en danger, ONED, 10 janvier 2007.
} 
Chaque fiche précise en outre les indicateurs spécifiques à l'étude :

- Processus relatifs à ceux précisés dans la littérature : rivalité, conflit, opposition, complicité, attachement, différenciation, etc.

- Phase développementale (0-5 ans, 6-12 ans, 13-18 ans)

- Mise à disposition de l'outil par les auteurs

- Validité de l'outil

- Durée de passation de l'outil

- Questionnaires avec réponses faites par l'enfant (avec ou sans aide de l'adulte) $)^{5}$.

Parmi les 21 outils recensés, 8 répondaient à l'ensemble de ces critères d'évaluation retenus pour la recherche, et 13 d'entre eux ont été écartés dans la mesure où ils ne répondaient pas à l'un ou l'autre (ou plusieurs) de ces critères ( $c f$. synthèse en annexe).

Au total, 8 questionnaires à questions fermées ont finalement été retenus pour être utilisés dans le cadre de la recherche-action. Ils sont présentés ci-dessous par ordre alphabétique :

1. Brother-Sister Questionnaire : BSQ1 (6-12 ans) et BSQ2 (> 12 ans) de Graham-Bermann et Cutler (1994)

2. Questionnaire des Relations Fraternelles: QRF1 de Troupel-Cremel (2006)

\footnotetext{
5 Compagnone et Lo Monaco (2015) soulignent l'importance de la prise en compte de la perception subjective des enfants du fonctionnement familial. Cette perception de l'enfant semble être un indice puissant renseignant sur la santé mentale future (Young \& al., 2011).
} 
3. Questionnaire des Relations Fraternelles: QRF2 de Hébert et Parent (1995)

4. Questionnaire des Relations Fraternelles: QRF3 de Claës, Poirier et Arseneault (2003)

5. Sibling Behaviors and Feelings Questionnaire : SBFQ de Mendelson, Aboud et Lanthier (1994)

6. Sibling Qualities Scale : SQS de Cole et Kerns (2001)

7. Sibling Relationship Inventory : SRI de Stocker et McHale (1992)

8. Sibling Relationships Questionnaire-Revised : le SRQ-R de Buhrmester et Furman (1990)

\subsection{Position éthique}

La passation s'est déroulée sous la responsabilité des psychologues de chacun des 12 villages.

Il a été décidé que :

- La passation se ferait à l'occasion d'un entretien prévu dans le cadre du suivi.

- Chaque passation serait réalisée impérativement par le psychologue.

- La lecture des items serait faite par les psychologues en fonction de l'âge des enfants et de leurs demandes éventuelles.

- L'information transmise à l'enfant, lors de la passation devait être la plus simple possible: expliquer que pour mieux accompagner les enfants, leur avis était important afin de connaitre les relations qui existaient avec leurs frères et 
sœurs. Il leur était également précisé qu'il n'y avait pas de bonne ou de mauvaise réponse, ni de jugement de valeur.

- Le résultat de la passation serait mis dans le dossier psychologique de l'enfant, accessible à l'équipe.

- Le libre choix a été laissé à l'enfant de répondre par rapport à tel ou tel membre de sa fratrie.

Chaque outil est accompagné de sa grille de cotation. L'outil était utilisé dans le cadre de la pratique courante pour éclairer les pratiques, ce n'est que secondairement que ce dispositif servait à la recherche.

\subsection{Présentation des outils}

Nous présentons par tranche d'âge chacun des 8 outils avec leurs objectifs, descriptifs et qualité psychométrique.

\subsubsection{Pour les 0-5 ans}

QRF 1 de Troupel-Cremel (2006): Questionnaire des Relations Fraternelles des jeunes enfants

Objectif : Déterminer les types de représentations des relations fraternelles selon deux axes, la coopération et l'opposition.

Description : 71 items interrogeant des domaines différents :

- les âges et sexes de la fratrie (4 items) ;

- la co-présence fraternelle (17 items) ; 
- la coopération fraternelle (26 items): se compose de 5 sous-échelles : sentiment, partage, tutelle, complicité et rôle parental

- l'opposition fraternelle (24 items) : comprend 3 sous-échelles : rivalité, différenciation et conflits.

Qualité psychométrique: La consistance interne du QRF1 est très bonne $\alpha=$ 0.689. Les alphas de Cronbach vont de 0.54 et 0.77 . La fiabilité test-retest des échelles, elle est très bonne dans la mesure où les CCI pour la coopération est de 0.868 , pour l'opposition de 0.79 et pour la coprésence de 0.87 .

SBFQ de Mendelson, Aboud et Lanthier (1994) : Sibling Behaviors and Feelings Questionnaire

Objectifs : Evaluer la qualité de la relation fraternelle de jeunes enfants en tenant compte des propres sentiments et comportements de l'enfant vis-à-vis de son F/S. Description : Il comporte 53 items reflétant 6 domaines :

- Sentiments positifs : admiration, affection, importance, satisfaction,

- Proximité : intimité, réconfort, sécurité,

- Conflit : antagonisme, compétition, domination, querelles,

- Identification : imitation et similarité,

- Soutien : aide et éducation,

- Amitié : temps passé ensemble, coopération, loyauté.

Qualité psychométrique: La consistance interne du SBFQ préscolaire est très bonne : les alphas de Cronbach vont de .75 à .87 après qu'un item ait été enlevé de l'échelle de conflit. La consistance interne des scores du SFBQ pour les enfants 
plus âgés va de «modérée » à « excellente » avec des alphas de Cronbach de .51 à .95 , après avoir de nouveau enlevé un item à l'échelle des conflits (Mendelson \& al., 1994).

\subsubsection{Pour les 6-12 ans}

SRQ-R de Buhrmester et Furman (1990): Sibling Relationships Questionnaire Revised

Objectif : Etablir l'influence qu'un enfant peut avoir sur son germain.

Description : il comporte 39 items mesurant 4 facteurs :

- Chaleur/Intimité : intimité, comportements prosociaux, similarité, admiration du F/S, admiration par le F/S, affection,

- Statut/pouvoir : soutien éducatif du F/S, domination du F/S

- Conflit : disputes, antagonisme et compétition,

- Rivalité : partialité maternelle et partialité paternelle.

Qualités psychométriques: Les indices de consistance interne (alpha de Cronbach) sont respectivement de $.71, .79, .77$, et .81 pour ces quatre souséchelles.

SRI de Stocker et McHale (1992) : Sibling Relationship Inventory

Objectifs : Evaluer la fréquence d'apparition des comportements et des sentiments dans l'interaction avec le frère ou la sœur.

Description : 20 items mesurent 3 domaines : 
- Chaleur/Affection: l'affection, l'admiration, l'enseignement, le soin, l'éducation et le partage.

- Rivalité : l'injustice parentale perçue et la jalousie envers l'attention parentale - Hostilité

Qualités psychométriques : Pour l'échelle Chaleur/Affection, 1'alpha de Cronbach est respectivement de .71 and .82 pour le cadet et l'aîné des enfants. Pour l'échelle Rivalité, l'alpha de Cronbach est respectivement de .86 pour l'aîné et de .89 pour le cadet. Pour l'échelle Hostilité, l'alpha de Cronbach est respectivement de .81 pour l’aîné et de .84 pour le cadet.

QRF 2 de Hébert et Parent (1995) : Questionnaire sur les relations fraternelles 2 $\underline{\text { Objectifs }}$ : Mesurer la qualité des relations de fratrie, les rapports qu'entretient un enfant avec un membre de sa fratrie.

Description : comporte 21 items permettant de mesurer 2 dimensions :

- Chaleur/Intimité : intimité, comportements prosociaux, similarité, affection - Statut/pouvoir : soutien éducatif du F/S.

Qualités psychométriques: Les indices de consistance interne (alpha de Cronbach) sont pour la version francophone (Hébert \& Parent, 1998) : pour la dimension « chaleur » : 0.89 et 0.61 pour la dimension « pouvoir ». Concernant la fiabilité test/retest (à 11.54 mois en moyenne), l'indice de stabilité est relativement élevé pour les dimensions chaleur/proximité $\left(\mathrm{r}_{\mathrm{s}}=0.69\right)$ et statut relatif/pouvoir $\left(r_{\mathrm{s}}=0.62\right)$. 
BSQ1 6-12 ans de Graham-Bermann et Cutler (1994): Brother-Sister

\section{Questionnaire}

Objectifs: Différencier les relations fonctionnelles des relations fraternelles dysfonctionnelles.

Description : C'est un questionnaire de 35 items mesurant 4 facteurs :

- Empathie: Cette échelle reflète le degré auquel les frères et sœurs sont émotionnellement liés et tournés vers ce que l'autre expérimente.

- Etablissement de frontières : Les six items de cette échelle évaluent le degré auquel les frères et sœurs sont en mesure de maintenir des frontières interpersonnelles.

- Similitude : Cette échelle de neuf items examine les intérêts et les expériences que les deux frères ont en commun.

- Pouvoir et contrôle : Ces six items évaluent le pouvoir et le contrôle que des F/S exercent l'un sur l'autre.

Qualités psychométriques: Cet outil a été validé sur les adolescents et jeunes adultes. Etant donné son intérêt au plan clinique et la facilité de compréhension de ses items, nous l'avons utilisé pour cette tranche d'âge 6-12 ans.

La consistance interne, signalée par les auteurs, est donnée par les Alpha de Cronbach suivants $:$ Empathie $=.92$, Frontières $=.85$, Similarités $=.73$, et Pouvoir et contrôle $=.69$. La fidélité test-retest sur une période de 10 jours et un échantillon de 25 étudiants est significative pour les 4 facteurs avec pour $p<.001$ Empathie $(r=.67)$, Frontières $(r=.65)$, Similarité $(r=.75)$ et Coercition $(r=$ .66). La fidélité pour l'échelle globale est de .91 pour l'ensemble des sujets. 


\subsubsection{Pour les $9-14$ ans}

SQS de Cole et Kerns (2001): Sibling Qualities Scale

Objectifs : Fournir une information globale sur le jugement qu'a l'enfant de la qualité de ses relations fraternelles.

Description: Il s'agit d'un auto-questionnaire de 43 questions mesurant six domaines :

- «Conflit et trahison» (7 items pour estimer le degré d'animosité et d'irrespect),

- «Résolution de conflit 》 (6 items pour estimer la résolution de conflit),

- «Aide et assistance» ( 9 items pour estimer l'aide apportée utile et bien intentionnée),

- «Soin et confiance en soi » (10 items pour estimer le sens apporté par le F/S aux sentiments et bien-être de son F/S),

- «Echanges intimes » (6 items pour estimer les sentiments intimes partagés),

- «Amitié » (5 items pour estimer dans quelle mesure l'enfant s'engage et s'amuse dans des activités avec son F/S).

Qualités psychométriques: Les alphas de Cronbach sont respectivement pour les 10,12 et 14 ans : «Conflit et trahison » (7 items) : .73, .79, .85; « Résolution de conflit $(6$ items $): .83, .89,87$; « Aide et assistance » $(9$ items $): .86, .87, .88$; «Soin et confiance en soi » (10 items) : .89, .76, .90; «Echanges intimes » (6 items) : .77, .83, .85 et «Amitié » (5 items) : .79, .83, .89. 


\subsubsection{Pour les plus de 12 ans}

QRF3 de Claës, Poirier et Arseneault (2003): Questionnaire sur les relations avec la fratrie

Objectifs : Mesurer la perception du jeune par rapport à la qualité de sa relation fraternelle.

Description: C'est un questionnaire de 20 items qui mesure trois principaux facteurs :

- La proximité (12 items) réfère aux sentiments subjectifs de proximité et à l'intimité vécue dans la relation fraternelle.

- Le conflit (4 items) réfère à la conviction d'être l'objet de critiques de sa part et de subir ses conduites guidées par la domination et la compétition.

- La rivalité (4 items) fait appel à des sentiments négatifs à l'égard du F/S et réfère au sentiment d'inégalité et d'injustice face au traitement des parents. Qualités psychométriques: L'étude de la validation a fait l'objet de plusieurs démarches de validation dans le cadre d'études portant sur le réseau social des adolescents (ex : Guichard, 1995). Une série d'analyses factorielles Varimax a permis d'identifier 3 facteurs identiques pour les deux versions du questionnaire, à savoir : 1) «proximité » $($ Cronbach/Paris $=.91), 2)$ « conflits » $($ Cronbach $=.65)$ et 3) «rivalité » $($ Cronbach $=.54)$. La structure factorielle est identique pour les deux versions, à l'exception de l'item 7 qui se retrouve dans le facteur «conflit» pour la version søur et dans le facteur «proximité » de la version frère. L'indice global de Cronbach est pour la version Frère de .92 et de .93 pour la sœur. 
BSQ2 > 12 ans de Graham-Bermann et Cutler (1994): Brother-Sister Questionnaire (cf. BSQ1)

\subsection{Description de l'échantillon testé}

L'échantillon se compose au total de 37 enfants accueillis au sein des 12 structures concernées. Chaque questionnaire a été testé auprès de 4 à 6 enfants répondant à la tranche d'âge concernée. Seul le BSQ2 > 12 ans de Graham et Cutler (1994) a été testé auprès de seulement deux enfants, et le SQS de Cole et Kerns (2001) a pu l'être auprès de 10 enfants de 9 à 14 ans.

Le nombre d'enfants, leurs âges moyens, la durée du placement des enfants et le temps de passation se répartissent de la façon suivante en fonction des tranches d'âge (Tableau I).

Insérer Tableau 1

Il est à noter que 211 fratries (soit 663 enfants) ont été accueillies au sein des 12 villages SOS en 10 ans entre 1996 et $2004^{6}$, soit une moyenne de 21 fratries (66 enfants tout âge confondu) par an, ce qui explique la taille réduite de l'échantillon testé.

\subsection{Critères d'évaluation retenus}

${ }^{6}$ http://www.sosve.org/images/pdf/ill_80_cahiersSOS2.pdf 
Les 8 outils retenus, les fiches de description des outils ${ }^{7}$ et les grilles d'analyse ${ }^{2}$ ont été présentés lors d'un séminaire de travail à l'association SOS Villages d'Enfants afin que les douze psychologues puissent poser les questions nécessaires et que les chercheurs soient certaines de la manière dont la passation se déroulerait. Après formation à la passation, il a ensuite été demandé, aux psychologues des 12 établissements de tester ces questionnaires dans leur pratique et de renseigner pour chacun d'entre eux une grille standardisée. Il s'agissait aussi de voir quelle pertinence chacun des outils avait à la fois dans le contexte du processus d'admission et dans celui de la prise en charge. Il s'agissait également d'avoir l'avis des psychologues sur la facilité d'utilisation de l'outil.

Cette grille a été bâtie selon les critères suivants :

- Critère 1 : objectifs visés par chacun des outils tels qu'ils ont été expérimentés par les psychologues

$\checkmark$ Aide à la compréhension des relations fraternelles

Aide à la décision du placement conjoint de la fratrie

Utilité comme complément à l'entretien lors de l'admission

Aide à l'appréciation du devenir des relations fraternelles

Intérêt des résultats pour la pratique

Fiabilité des résultats

- Critère 2 : forme (clarté des questions, des consignes; adapté à l'âge des enfants ; utilisable en l'état sans modification ; facilité de passation)

${ }^{7}$ Disponibles auprès du premier auteur 
- Critère 3 : utilité de la passation de l'outil lors de l'admission de l'enfant dans la structure $^{8}$

- Critère 4 : facilité d'interprétation des résultats

- Critère 5 : utilité pour évaluer l'évolution des relations fraternelles au cours du placement

Les scores pour chaque critère étaient notés selon une échelle de Likert en 4 points. ( 0 : pas satisfait $; 1$ : peu satisfait $; 2$ : satisfait $; 3$ : totalement satisfait).

\section{Résultats de l'évaluation}

Les scores pour chacun des cinq indicateurs, apparaissant dans le tableau II, sont obtenus en calculant la moyenne de satisfaction obtenue pour chaque outil sur l'échelle de Likert en 4 points ( 0 : pas satisfait ; 1 : peu satisfait ; 2 : satisfait ; 3 : totalement satisfait).

Les psychologues ne sont pas satisfaits si le score est strictement inférieur à 1 , moyennement satisfaits s'il se situe entre 1 et 1.4 , satisfaits entre 1.5 et 2 , et très satisfaits si le score est supérieur à 2 .

Insérer Tableau 2

\subsection{Critère 1 : Objectifs visés pas le questionnaire}

\footnotetext{
${ }^{8}$ Selon Dumaret et Crost (2008), le repérage des dysfonctionnements doit avoir lieu dès l'admission en placement ou dans les mois qui suivent afin de mettre en place au plus vite des soins adaptés.
} 
Les résultats indiquent que les scores obtenus pour les trois outils que sont le SQS (9-14 ans), QRF3 (> 12 ans), et le BSQ2 > 12 ans sont satisfaisants, et ne le sont que moyennement ou très peu pour les 5 autres outils.

\subsection{Critère 2 : Forme des questionnaires}

Les outils pour les plus jeunes (0-5 ans), que sont le QRF1 et le SBQF de même que le QRF2 (6-12 ans), sont les moins satisfaisants, puis viennent les SRI (6-12 ans), SRQ (6-12 ans) et QRF3 (> 12 ans) qui apparaissent satisfaisants. Les outils tels que le BSQ1 (6-12 ans), SQS (9-14 ans) et le BSQ2 > 12 ans sont très satisfaisants, notamment le SQS pour les 9-14 ans.

\subsection{Critère 3 : Utilisation lors de l'admission de l'enfant}

La quasi-totalité des questionnaires (7/8) ne semble pas satisfaisante pour l'utilisation lors de la première rencontre entre l'enfant et le psychologue de la structure. Seul le BSQ2 > 12 ans apparaît utilisable dès la première rencontre avec le jeune.

\subsection{Critère 4 : Facilité d'interprétation des résultats}

Les scores sont compris entre 1.5 (satisfaisant) et 2.4 (très satisfaisant). Ainsi, les questionnaires testés ne posent pas problème aux professionnels pour être cotés et interprétés. 


\author{
3.5. Critère 5 : Utilisation pour évaluer l'évolution des relations fraternelles \\ Plusieurs questionnaires paraissent pertinents afin de pouvoir évaluer l'évolution \\ des relations fraternelles : le QRF 1 (0-5 ans), les QRF2 et SRQ pour les 6-12 ans, \\ le SQS pour les 9-14 ans et enfin le QRF3 pour les enfants de plus de 12 ans. Ne \\ répondent pas à cet objectif les questionnaires SBFQ (0-5 ans), SRI (6-12 ans), \\ BSQ1 6-12 ans et BSQ2 > 12 ans.
}

\title{
3.6. Synthèse des résultats
}

Les résultats que nous avons obtenus quant à l'évaluation faite par les psychologues des douze Villages d'enfants peuvent se synthétiser de la manière suivante :

- Il apparaît que les outils sont d'autant plus utiles aux psychologues pour évaluer les relations fraternelles qu'ils s'adressent à des enfants d'au moins 6 ans. Si l'on considère, de surcroit, le critère « objectifs visés par l'outil », trois questionnaires émergent, SQS 9-14 ans, le QRF 3 (> 12 ans) et le BSQ2 > 12 ans. Autrement dit, c'est seulement à partir de 9 ans que les outils recensés sont pleinement utilisables par les professionnels tout en étant renseignés par les enfants eux-mêmes. Les psychologues ont estimé que ces outils constituaient des compléments précieux aux rencontres plus institutionnelles pour appréhender la qualité des relations fraternelles, et pour leur permettre d'envisager au mieux l'utilisation des ressources fraternelles quand elles 
existent et de protéger l'enfant des maltraitances entre enfants quand elles adviennent (Dayan, Pinel-Jacquemin \& Scelles, 2011).

- C'est le BSQ2 > 12 ans $^{9}$ qui obtient le plus de satisfaction sur la quasi-totalité des indicateurs évalués, en dehors de son utilisation à plusieurs reprises au cours du placement.

- Concernant l'évaluation des relations fraternelles en cours de placement, les QRF1, QRF2, SRQ-R, SQS et QRF3 sont les cinq outils qui pourraient être une aide précieuse aux professionnels pour conforter (ou non) le placement conjoint des fratries.

- Les questionnaires à visée de recherche qui ont été renseignés par les psychologues des 12 Villages sont tous facilement utilisables dans le cadre de leur pratique professionnelle.

\section{Discussion}

L'échantillon restreint des 37 enfants concernés par cette étude et le fait que les données aient été recueillies au sein des établissements d'une seule association partenaire invitent évidemment à la plus grande prudence concernant la portée de nos conclusions.

La méthodologie de cette recherche-action a permis d'identifier les questionnaires les plus pertinents dans le cadre d'une pratique professionnelle et ce, en fonction des différentes périodes développementales des enfants, ce

\footnotetext{
${ }^{9}$ Nous rappelons cependant qu'il n'a été renseigné que par deux enfants.
} 
qui ouvre des pistes de réflexions quant à leur utilisation et la manière de travailler sur et avec la fratrie en fonction de l'âge des enfants.

Par-delà ce premier objectif visé, cette recherche-action a permis d'accompagner les équipes dans leur travail de réflexion avec les fratries au sein des villages. Elle a conduit les professionnels à prendre conscience de l'intérêt de se doter d'outils aidant à mieux évaluer la nature et la dynamique des liens fraternels, et de prendre en compte le point de vue des enfants.

L'intérêt que les professionnels et l'association gestionnaire a porté à cette étude démontre une demande forte des acteurs de terrain, et en particulier, des psychologues qui ont besoin de se doter d'outils à même de les aider à éclairer les équipes sur ce qui se vit au sein des fratries : pour évaluer les fonctions soutenantes ou toxiques des liens entre enfants dans la cadre d'une décision de placement; repérer et prendre en compte les éventuelles maltraitances entre enfants avant et durant le placement.

Cette recherche-action a contribué à la fois à sensibiliser l'ensemble des villages à l'importance de mieux comprendre et d'évaluer ce qui se passe entre les enfants. Ainsi, si ce sont les psychologues qui ont «testé » les outils, cette utilisation s'est faite dans le cadre d'un travail d'équipe qui a impliqué l'ensemble du village SOS. Ainsi, cette recherche-action, par-delà son intérêt pour la recherche a eu un effet de formation, d'information mais aussi de mobilisation des villages concernant les liens fraternels. Les villages SOS ont témoigné du fait, que cette expérience a favorisé le dialogue entre 
professionnels et a fait naître chez ces derniers, et pas seulement les psychologues, des demandes de formations complémentaires.

Cette expérience à laquelle des psychologues ont été étroitement associés montre la nécessité de poursuivre des travaux pour que les qualités de liens fraternels soient mieux prises en compte, ce qui passe par des protocoles validés scientifiquement visant à les évaluer.

Cette recherche-action montre sans contexte, l'intérêt que de manière rigoureuse, le chercheur puisse recueillir et analyser le point de vue de l'usage des outils par les professionnels/psychologues pour formuler ses recommandations concernant les limites et intérêts des outils disponibles.

Durant ces études, avec leurs collègues, mais également avec les chercheurs, les psychologues ont eu l'occasion de formuler clairement les questions qu'ils se posaient, d'imaginer comment y répondre. Ils ont ainsi à la fois lors de la formation initiale et lors de la restitution, formalisé individuellement et collectivement leurs besoins, souhaits en matière d'évaluation des liens fraternels.

Durant cette recherche-action, les chercheurs ont pu expérimenter tout l'intérêt du transfert des outils de recherche au sein d'institutions, ce qui construit un pont entre le monde académique et la sphère professionnelle source d'enrichissement mutuel. Elle a permis aussi aux professionnels, comme aux chercheurs, de mesurer la tension qu'il peut y avoir entre une question urgente dans la cadre de la pratique et les difficultés à y répondre simplement. Les liens fraternels nécessitent une approche complexe, pour laquelle les outils peuvent 
aider, mais ils resteront un support indispensable qui doit s'accompagner d'une approche impliquant l'écoute et l'observation fine des enfants.

En conclusion, il apparaît que certains outils sont manifestement utiles et utilisables dans la pratique professionnelle. Par rapport aux questions que nous avons posées dans notre introduction, leur mise à l'épreuve par les psychologues eux-mêmes a permis d'amener des pistes de réflexion sur ce qu'est une fratrie, sur la qualité de la relation fraternelle, pour éventuellement modifier les regards, affiner les savoirs, voire modifier les pratiques sur la conduite à tenir quant à la séparation ou non des fratries et, dans chacun des cas, sur quels critères.

Tout comme le stipulent «Les chercheurs ignorants » (2015), cette rechercheaction menée par des chercheurs et des acteurs de terrain, en collaboration étroite, à distance des approches académiques classiques, participe à améliorer nos connaissances afin de mieux prendre en compte la dimension fraternelle et ses avatars quand il est question de séparer ou non les fratries. Pour autant, cette étude exploratoire indique également que d'autres outils restent donc à concevoir pour améliorer l'objectivation de la (dys) fonctionnalité des relations fraternelles, en particulier auprès des jeunes enfants. Elle ouvre, enfin, des pistes qui pourraient être poursuivies pour mener un travail de plus grande envergure au sein d'autres institutions que celle dans laquelle la recherche-action s'est déroulée tant au plan national qu'au niveau international. 
1

2

3

4

5

6

7

8

10

11

12

13

14

15

16

17

18

19

20

21

22

23

24

25

26

27

28

29

30

31

32

33

34

35

36

37

38

39

40

41

42

43

44

45

46

47

48

49

50

51

52

53

54

55

56

57

58

59

60

61

62

63

64

65

Conflit d'intérêt : aucun 
Bibliographie

Adler, A., 1920. Pratique et théorie de la psychologie individuelle. Paris : Payot, 1958.

Angel, S., 1996. Des frères et des sœurs: les liens complexes de la fraternité, Paris, Robert Laffont.

Arranz, E., Olabarrieta, F., Yenes, F., \& Martin, J., 2001. Perceptions of sibling relationships in children aged 8 to 11 . Revista De Psicologıa General y Aplicada $54,425-441$.

Bass, L. B., Taylor, B. A., Knudson-Martin, C., Huenergardt, D., 2006. Making Sense of Abuse: Case Study in Sibling Incest. Contemporary Family Therapy: An International Journal 28(1), 87-109.

Beauregard, K., 2003. Qualité de la relation fraternelle et adaptation des frères et sœurs placés conjointement ou séparément en famille d'accueil. Thèse de doctorat, sous la direction du Pr. Daniel Paquette, Université de Montréal.

Benarous, X., Consoli, A., Raffin, M. et Cohen, D., 2014. Abus, maltraitance et négligence: (1) épidémiologie et retentissements psychiques, somatiques et sociaux. Neuropsychiatrie de l'enfance et de l'adolescence 62, 299-312.

Bouhassira Chiron, M., 2012. Frères et sæurs intimes ennemis : à propos du complexe fraternel. Paris : l'Harmattan.

Bourguignon, O., 1999. Le fraternel. Paris : Dunod.

Buhrmester, D., \& Furman, W., 1985. Children's perceptions of the personal relationships in their social networks. Developmental Psychology 21(6), 10161024. 
Buhrmester, D., \& Furman, W., 1990. Perceptions of sibling relationships during middle childhood and adolescence. Preview. Child Development 61(5), 13871398.

Candoret, A., 1998. Placer ensemble les frères et sœurs ? Informations sociales, Frères et sœurs 67, 74-81.

Claës, M., Poirier., \& Arseneault, M. J., 2003. Le Questionnaire sur les relations avec la fratrie. Document interne. Montréal. Université de Montréal. Département de Psychologie.

Cole, A. K., \& Kerns, K. A., 2001. Perceptions of sibling qualities and activities of early adolescents. Journal of Early Adolescence 21, 204-227.

Compagnone, P. \& Lo Monaco, G., 2015.Validation française du questionnaire d'évaluation de l'environnement invalidant durant l'enfance: The Invalidating Childhood Environments Scale (ICES). Revue Européenne de Psychologie Appliquée 65 (1), 43-52.

Daniels, D. \& Plomin, R., 1985. Differential experience of siblings in the same family. Dev Psychology 21, 747-760.

Dayan, C., Pinel-Jacquemin, S., \& Scelles, R., 2011. Le placement conjoint de fratries : regards croisés de professionnels sur leur pratique. Bulletin de Psychologie 64(4), 329-337.

Dunn, J., 1983. Sibling relationships in early childhood. Child Development 54(4), 787-811. 
Dumaret, A-C. \& Crost, M., 2008. Placements à long terme en villages d'enfants depuis plus de 30 ans : troubles psychiques et prise en charge. Neuropsychiatrie de l'enfance et de l'adolescence 56, 512-519.

Drapeau, S., Simard, M., Beaudry, M. \& Charbonneau, C., 2000. Siblings in family transitions, family relations. Interdisciplinary Journal of Applied Family Study 49 (1). $77-85$.

Fine, A., 2007. Les fratries en Europe. Aperçu sur quelques orientations de recherche en anthropologie. In M. Oris, G. Brunet, E. Widmer, \& A. Bideau, Les Fratries. Une démographie sociale de la germanité ? Berne : Peter Lang, pp. 4778.

Freud S., 1922. Sur quelques mécanismes névrotiques dans la jalousie, la paranoïa et 1'homosexualité, trad. fr., in : Névrose, psychose et perversion. Paris : P.U.F., 1973, pp. 271-281.

Graham-Bermann, S. A., 1994. The assessment of childhood sibling relationships: varying perspectives on cooperation and conflict. The Journal of Genetic Psychology 155(4), 457-469.

Graham-Bermann, S. A. \& Cutler, S. E., 1994. The Brother-Sister Questionnaire: Psychometric Assessment and Discrimination of Well-Functioning From Dysfunctional Relationships. Journal of Family Psychology 8(2), 224-238.

Guichard, A., 1995. Fratrie, qualité de la relation fraternelle et ajustement psychologique au cours de l'adolescence. Comparaison France-Québec. Mémoire de maîtrise inédit. Montréal : Université de Montréal. Département de Psychologie. 
Hébert, M., \& Parent, N., 1995. Traduction française du Sibling Relationships Questionnaire de D. Buhrmester, \& W. Furnam (SRQ; 1985). Ste-Foy, QC : Université Laval.

Hébert, M., \& Parent, N., 1998. Psychometric properties of the French version of the Sibling Relationships Questionnaire. Département d'Orientation, d'Administration et d'Evaluation, Université Laval.

Jaïtin, R., 2006. Clinique de l'inceste fraternel. Paris : Dunod

Les «chercheurs ignorants », (dir.), 2015. Les recherches-actions collaboratives. Une révolution de la connaissance, Rennes, Presses de l'EHESP.

Kaës, R., 1993. Le complexe fraternel, aspect de sa spécificité. Topique, 51, 5-43.

Kramer, L., \& Baron, L. A. (1995). Parental perceptions of children's sibling relationships. Journal of Applied Family and Child Studies 44, 95-103.

Mendelson, M. J., Aboud, F. E., \& Lanthier, R. P., 1994. Kindergartner's relationships with siblings, peers, and friends. Merrill-Palmer Quarterly 40(3), 416-435.

Mendelson, M. J., Aboud, F. E., \& Lanthier, R. P., 1994. Kindergartner's relationships with siblings, peers, and friends. Merrill-Palmer Quarterly 40(3), 416-435.

Minuchin, S., 1979. Familles en thérapies. Paris : Jean-Pierre Delarge.

Pinel-Jacquemin, S., Chéron, J., Favart, E.., Dayan, C., \& Scelles, R., 2013. Sibling Violence and joint placement: a review of the literature. Early Child Development and Care 183(7), 963-980. 
Pinel-Jacquemin, S., \& Scelles, R., 2012. La violence fraternelle existe : ne la banalisons pas ! Revue Québécoise de Psychologie 33(3), 187-212.

Rabain J. F., 1995. La rivalité fraternelle. In S. Lebovici, R. Diatkine, M. Soulé (dir.), Nouveau traité de psychiatrie de l'enfant et de l'adolescent, 2e éd. Paris : P.U.F, pp. 2549-2576.

Riggio, H. R., 2000. Measuring attitudes toward adult sibling relationships: The Lifespan Sibling Relationship Scale. Journal of Social and Personal Relationships 17(6), 707-728.

Ross, H. S., Woody, E., Smith, M., \& Lollis, S., 2000. Young children's appraisals of sibling relationships. Merrill-Palmer Quarterly 46, 441-464.

Rufo, M., 2003. Frères et sœurs, une maladie d'amour. Paris : Fayard.

Sharifi, T., Jabbarifard, F., Rezaee, A. M., Hafshejani, S. F. \& Zare, M., 2015. The Impact of Temperament and Character on the Quality of Sibling Relationships. MAGNT Research Report 3(3), 1395-1405.

Scelles, R., 2003. Frères et sœurs, complices et rivaux. Paris : Fleurus.

Scelles, R., 2010. Liens fraternels et handicap. Toulouse : Erès.

Scelles, R., Favart, E., Chéron, J., \& Pinel-Jacquemin, S., 2008. Accueil conjoint des fratries. Analyse de la littérature en sciences humaines et sociales. Rapport Terminal, SOS Villages d'Enfants et Fondation de France, Septembre 2008.

Schaeffer, E.S. \& Edgerton, M., 1981. The Sibling Inventory of Behavior. Chapel Hill, University of North Carolina.

Simard, M., Beaudry, M., Drapeau, S., \& Charbonneau, C., 1998. Les changements dans le système fraternel chez les enfants placés en milieu 
substitut. Dans : L. S. Ethier et J. Alary (Eds). Comprendre la famille. Acte du 4ème symposium québécois de recherche sur la famille. Ste-Foy, Qc.

Slomkowski, C., Wasserman, G., \& Schaffer, D., 1997. A new instrument to assess sibling relationships in antisocial youth: The Social Interaction Between Siblings (SIBS) Interview: A research note. Journal of Child Psychology and Psychiatry 38(2), 253-256.

Stocker, C., Dunn, J., \& Plomin, R., 1989. Sibling relationships: Links with child temperament, maternal behavior, and family structure. Child Development 60, $715-727$.

Stocker, C., \& McHale, S., 1992. The nature and family correlates of preadolescents' perceptions of their sibling relationships. Journal of Personal and Social Relationships 9, 179-195.

Strauss, M. A., 1979. Measuring intrafamily conflict and violence: The conflict tactics (CT) scales. Journal of Marriage and the Family 41, 75-88.

Tilmans-Ostyn, E., \& Meynckens-Fourez, M. (Eds), 1999. Les Ressources de la Fratrie. Toulouse : Erès.

Troupel-Cremel, O., 2006. Attachement fraternel, styles des relations et des interactions de tutelle au sein des fratries de jeunes enfants : effet modulateur de la représentation des relations fraternelles de l'aîné. Thèse de doctorat, sous la direction du Pr. Chantal Zaouche-Gaudron, Université Toulouse II-Le Mirail, UFR de Psychologie - Département de Psychologie du développement. 
Troupel-Cremel, O., Pinel-Jacquemin, S., \& Zaouche-Gaudron, C., 2009. Fratrie et Violence intrafamiliale: la fratrie obstacle ou ressource pour l'enfant ? Archives de Pédiatrie 16(6), 943-945.

Vallerand, R. J., 1989. Vers une méthodologie de validation transculturelle de questionnaires psychologiques : implications pour la recherche en langue française. Psychologie Canadienne 30 (4), 662-689.

Vinay, A. \& Jayle, S., 2011. Faire Fratrie: réflexions autour du lien fraternel. Neuropsychiatrie de l'enfance et de l'adolescence 59, 342-347.

Volling, B. L., 1997. The family correlates of maternal and paternal perceptions of differential treatment in early childhood. Family Relations 46, 227-236.

Volling, B.L., Youngblade, L.M. \& Belsky, J., 1997. Young children's social relationships with siblings and friends. American Journal of Orthopsychiatry 67(1), 102-111.

Widmer, E., 1999. Les relations fraternelles des adolescents. PUF : Paris.

Young, R., Lennie, S. \& Minnis, H., 2011. Children's perceptions of parental emotional neglect and control and psychology. Journal of Child Psychology and Psychiatry 52, 889-897.

Zaouche-Gaudron, C., Euillet, S., \& Pinel-Jacquemin, S., 2007. Recensement critique des instruments de recherche validés en langue française en psychologie du développement : Bilan sur 10 ans (1995 à 2005). Rapport terminal, Observatoire National de l'Enfance en danger, ONED, 10 janvier 2007.

Zaouche Gaudron, C., Troupel-Cremel, O., \& Pinel-Jacquemin, S., 2010. Améliorer l'accompagnement des enfants pendant le placement : pour une 
1

2

meilleure analyse de la dimension fraternelle. Rapport terminal, SOS Villages d'Enfants et Observatoire National de l'Enfance en Danger, Octobre 2010. 


\section{Synthèse des outils non retenus}

13 outils ne répondaient pas à l'un ou l'autre (ou plusieurs) des critères retenus :

\section{Processus mesurés:}

- Questionnaire on Sibling Relationships d'Arranz \& al., 2001 : évalue la satisfaction de l'enfant $\underline{\text { de sa place dans la fratrie et non la qualité de la relation fraternelle elle-même. }}$

- Social Interaction Between Siblings de Slomkowski \& al., 1997 : évalue la relation fraternelle dans un contexte de délinquance.

- Sibling Inventory of Differential Experience (S.I.D.E.) de Daniels et Plomin, 1985 : compare les $\underline{\text { expériences de l'enfant à celles de ses frères et sœurs dans le domaine du traitement parental, de }}$ l'interaction fraternelle et des caractéristiques des pairs.

- Sibling Inventory Behavior (S.I.B.) de Schaeffer et Edgerton, 1981 : cet outil a été conçu à l'origine pour les fratries comprenant un enfant handicapé.

- Conflict Tactics Scale (CTS) de Straus, 1979: ce questionnaire est uniquement axé sur les conflits.

- Lifespan Sibling Relationship Scale (L.S.R.S.) de Riggio, 2000 : interroge l'adulte sur ses relations fraternelles dans l'enfance.

Mise à disposition de l'outil par les auteurs - Sibling Relationships in Early Childhood questionnaire de Volling, 1997 : cet outil n'a pu être obtenu.

Validité de l'outil (insuffisante ou inconnue):

Le Questionnaire des relations fraternelles des adolescents et des jeunes adultes de Widmer, 1999: cet outil n'a pas fait l'objet d'une validation ad hoc, le Sibling Relationship Questionnaire de Ross, 2000 est un outil projectif, sans cotation, et non validé, de même le Sibling Story Test de Graham-Bermann (1994) est un test projectif sans possibilité de cotation.

Durée de passation trop longue

- Observation des relations fraternelles de Volling \& al., 1997 : cet outil nécessitant une visite à $\underline{\text { domicile de deux heures, il ne pouvait rentrer dans le cadre de notre recherche-action au sein des }}$ villages d'enfants.
Formatted: Font: Not Bold

Formatted: Font: $10 \mathrm{pt}$

Formatted: Font: $10 \mathrm{pt}$

Formatted: Font: $10 \mathrm{pt}$

Formatted: Font: 10 pt, Not Highlight

Formatted: Font: $10 \mathrm{pt}$, Not Highlight

Formatted: Font: 10 pt, Not Highlight

Formatted: Font: $10 \mathrm{pt}$, Not Highlight 
Formatted: Font: $10 \mathrm{pt}$

Formatted: Font: $10 \mathrm{pt}$, Not Bold 
Tableau I : Répartition des outils et des enfants testés selon l'âge moyen et la durée de placement

\begin{tabular}{|c|c|c|c|c|}
\hline $\begin{array}{l}\text { Tranche } \\
\text { D'âge }\end{array}$ & & $\begin{array}{c}\text { Nombre } \\
\text { d'enfants testés }\end{array}$ & $\begin{array}{l}\text { Age moyen des } \\
\text { enfants (ans) }\end{array}$ & $\begin{array}{l}\text { Durée du placement } \\
\text { (ans) }\end{array}$ \\
\hline $0-5$ ans & $\begin{array}{l}\text { QRF1 (Troupel-Cremel, 2006) } \\
\text { SBFQ (Mendelson \& al., 1994) }\end{array}$ & $\begin{array}{l}4 \\
5\end{array}$ & 5,2 & 1,8 \\
\hline 6-12 ans & $\begin{array}{l}\text { QRF2 (Hébert \& Parent, 1995) } \\
\text { SRI (Stocker \& McHale, 1992) } \\
\text { SRQ-R (Buhrmester \& } \\
\text { Furman, 1990) } \\
\text { BSQ1 (Graham \& Cutler, } \\
\text { 1994) }\end{array}$ & $\begin{array}{l}5 \\
5 \\
6 \\
5\end{array}$ & 9,13 & 3,32 \\
\hline 9-14 ans & SQS (Cole \& Kerns, 2001) & 10 & 11,9 & 4,88 \\
\hline$>12$ ans & $\begin{array}{l}\text { QRF3 (Claës \& al. 2003) } \\
\text { BSQ2 > } 12 \text { ans (Graham \& } \\
\text { Cutler, 1994) }\end{array}$ & 5 & 14,85 & 6,7 \\
\hline
\end{tabular}


Tableau II : Temps moyen de passation par tranches d'âge

\begin{tabular}{|c|l|c|}
\hline \multirow{2}{*}{$\begin{array}{c}\text { Tranche } \\
\text { d'âge }\end{array}$} & \multicolumn{1}{|c|}{ Outils } & $\begin{array}{c}\text { Temps moyen de passation } \\
\text { (min) }\end{array}$ \\
\hline \multirow{2}{*}{$\mathbf{0 - 5}$ ans } & SBFQ & 13.5 \\
\cline { 2 - 3 } & QRF1 & 14.38 \\
\hline \multirow{4}{*}{ 6-12 ans } & SRQ-R & 22.25 \\
\cline { 2 - 3 } & BSQ 6-12 & 13.3 \\
\cline { 2 - 3 } & & 14.38 \\
\cline { 2 - 3 } & SRI & 12 \\
\hline \multirow{2}{*}{ 9-14 ans } & SQS & 19.56 \\
\hline \multirow{2}{*}{$\mathbf{1 2}$ ans } & QRF3 & 7.5 \\
\cline { 2 - 3 } & BSQ > 12 & 9.5 \\
\hline
\end{tabular}


Cher-e-s collègues,

Nous remercions les experts pour leurs remarques constructives qui nous ont permis d'améliorer notre document.

Toutes les remarques de forme, de fond, de plan ont été prises en compte. Nous les détaillons ci-dessous.

En espérant que cette révision répondra à vos attentes.

Bien à vous

Thématique tout à fait intéressante et nécessaire dans la pratique psychologique autour des fratries. L'article apporte une réflexion méthodologique utile et pointe la nécessité d'une réflexion autour des liens fraternels et des pratiques de placement. Toutefois, quelques éléments de présentation et de conception de l'article nous semblent à améliorer. L'introduction pose une problématique évidente et intéressante à propos des décisions de placement, des liens fraternels, des relations dans la fratrie et de leurs enjeux à des niveaux conscient et inconscient. Dans cette annonce prometteuse, la discussion et la conclusion de l'article apportent essentiellement une réponse méthodologique. L'article peut être nettement amélioré avec un effort de liaison entre l'amorce de réflexion du début et les pistes issues de la recherche-action.

Nous avons modifié la discussion et la conclusion en ce sens afin d'établir un lien plus étroit entre les questions posées dans l'introduction et les résultats issus de cette recherche-action.

Ainsi après l'étude approfondie de 8 outils, comment peut-on mieux accompagner le placement des fratries! ? Comment peut-on évaluer les situations de maltraitance fraternelle ? Comment les professionnels, grâce à ces outils, peuvent modifier leur champ d'action auprès des fratries ?...

Page 3, ligne 53-54 : outils d'évaluation spécifiques et adaptées $=>$ Outils d'évaluation spécifiques et adaptés. FAIT

Page 4, ligne 19 : «ou doivent être séparés » : préciser les raisons d'un devoir de séparation.

Nous avons, dans l'introduction » amener les raisons qui amènent ou non à une séparation de la fratrie et pris en compte les pistes de réflexion proposées.

Page 5, ligne 2 : "versus" en italique : FAIT

Page 5, lignes 45-54 : d'abord étudié... (références Freud, 1922 ; Rabain, 1995) à présent... (Freud 1922 ; Rabain, 1995) : une chronologie est présentée au niveau de l'intérêt de recherche mais les références citées restent identiques et de plus, peu d'actualité : 1922, 1995. FAIT 
Page 6, ligne 12 : phénomènes complexes "d'indentification" : FAIT

Page 9, ligne 7 : espace entre projection/ introjection FAIT

Pages 11 à 13 : le listing des 13 outils écartés est-il nécessaire ici ? les raisons ayant amené à écarter ces outils paraissent intéressantes, mais trois pages à détailler des outils qui ne seront pas étudiés et qui ont vraisemblablement déjà fait l'objet d'une publication, est-ce bien utile ici ? Ou alors une présentation sous forme de tableau plus synthétique pourrait être intéressante. D'autant plus qu'on apprend les raisons pour lesquelles ils n'ont pas été retenus, mais pas celles pour lesquelles les 8 autres l'ont été.

La synthèse a été mise en annexe

Page 14, ligne 7 : le 8 . semble barré. FAIT

Page 14, ligne 30 : chaque passation se ferait $=>$ chaque passation serait réalisée FAIT

Page 14, lignes 40-43 : répétition "pour mieux" FAIT

Page 26, Discussion : reformulation du paragraphe, il ne s'agit pas ici encore de la discussion mais plutôt des résultats. Le titre « résultats » paraît plus correct ici.

Le plan a été modifié en prenant en compte cette remarque.

Page 27, ligne 29 : La méthodologie.... Cette partie me semble davantage correspondre à une discussion, qui pourrait être encore étayée sur les principes fondamentaux de la recherche-action (cf. Collectif « Les chercheurs ignorants » (2015). La recherche-action collaborative : une révolution de la connaissance. Paris : EHESP), sur ce que cette démarche a généré auprès des professionnels mais aussi et surtout sur la prise de décision au placement en fratrie ou non (problématique de départ de l'article). Dans le résumé il est indiqué que cette RA a eu pour visée de contribuer à la prise en considération de la dimension fraternelle par les professionnels : dans la discussion pourrait davantage apparaître cette prise en considération, comment cette RA a modifié les regards, les savoirs et les pratiques?

Les propositions ont été prises en compte et nous avons repris le plan en fonction de cette remarque.

Page 28, ligne 49 : Conclusion, peut-être à étoffer un peu comme par exemple, davantage de liens entre la problématique posée dans l'introduction et les conclusions de cette RA.

Nous avons étoffé la conclusion afin de répondre à cette remarque. 